\title{
The utility of a prognostic index for predicting time to first treatment in early chronic lymphocytic leukemia: the GIMEMA experience
}

\author{
Stefano Molica, ${ }^{1}$ Francesca R. Mauro, ${ }^{2}$ Vincenzo Callea, ${ }^{3}$ Diana Giannarelli, ${ }^{4}$ Francesco Lauria, ${ }^{5}$ Bruno Rotoli, ${ }^{6}$ \\ Agostino Cortelezzi, ${ }^{7}$ Vincenzo Liso, ${ }^{8}$ and Robin Foà ${ }^{2}$
}

${ }^{1}$ Department of Hematology-Oncology, Azienda Ospedaliera Pugliese-Ciaccio, Catanzaro, Italy; ${ }^{2}$ Department of Cellular Biotechnologies and Hematology, Division of Hematology, Sapienza University, Rome, Italy; ${ }^{3}$ Department of Hematology, Ospedali Riuniti, Reggio di Calabria, Italy; ${ }^{4}$ Biostatistics, Regina Elena National Cancer Institute, Rome, Italy; ${ }^{5}$ Department of Hematology and Transplant, University of Siena, AOUS, Siena, Italy; ${ }^{6} \mathrm{Hematology}$ Unit, Federico II University, Naples, Italy; ${ }^{7} \mathrm{Hematology-Bone}$ Marrow Transplant Unit, Fondazione Ospedale Maggiore Maggiore Policlinico, Mangiagalli, Regina Elena IRCCS, Milano, Italy, and ${ }^{8}$ Hematology Section, DAP, University of Bari, Italy

\section{ABSTRACT}

\section{Background}

A prognostic index based on widely available clinical and laboratory features was recently proposed to predict survival in patients with previously untreated chronic lymphocytic leukemia. We assessed the utility of this index for predicting time to first treatment in early chronic lymphocytic leukemia.

\section{Design and Methods}

An observational database of the GIMEMA (Gruppo Italiano Malattie EMatologiche dell'Adulto), which included 310 patients with newly diagnosed Binet stage A chronic lymphocytic leukemia who were observed at different primary hematology centers during the period 1991 -2000 , was used for the purpose of this study.

\section{Results}

The new prognostic index enabled Binet stage A patients to be divided into two subgroups that differed with respect to time to first treatment $(P=0.003)$. The original prognostic index was derived from a database that included cases observed at a reference academic center; these patients were younger $(P<0.0001)$ and had more advanced disease $(P<0.0001)$ than those in the current investigation, which studied community-based patients whose data were recorded at presentation. With this in mind, we used an optimal cut-off search to determine how best to split patients with Binet stage A disease into different prognostic groups. According to the recursive partitioning (RPART) model, a classification tree was built that identified three subsets of patients who scores were 0-2 (low risk), 3-4 (intermediate risk) and 5-7 (high risk). The probability of remaining free from therapy at 5 years was $100 \%$ in the low risk group, $81.2 \%$ in the intermediate risk group and $61.3 \%$ in the high risk group $(P<0.0001)$.

\section{Conclusions}

The results of this study confirm the utility of a new prognostic index for predicting time to first treatment in a large sample series of community-based patients with early stage chronic lymphocytic leukemia at presentation. Our effort to develop a revised scoring method meets the need to separate Binet stage A patients into different prognostic groups in order to devise individualized and tailored follow-up during the treatment-free period.

Key words: prognostic index, early chronic lymphocytic leukemia, disease progression.

Citation: Molica S, Mauro FR, Callea V, Giannarelli D, Lauria F, Rotoli B, Cortelezzi A, Liso V, and Foà $R$ on behalf of the GIMEMA CLL study group. The utility of a prognostic index for predicting time to first treatment in early chronic lymphocytic leukemia: the GIMEMA experience. Haematologica. 2010; 95:464-469. doi: 10.3324/haematol.2009.011767

(C)2010 Ferrata Storti Foundation. This is an open-access paper.
Manuscript received May 20, 2009. Revised version arrived September 7, 2009. Manuscript accepted September 9, 2009.

Correspondence:

Stefano Molica, MD,

Dept. Hematology/Oncology,

Azienda Ospedaliera

Pugliese-Ciaccio, Viale Pio X

88100 Catanzaro, Italy.

Email: smolica@libero.it 


\section{Introduction}

In parallel with significant improvements in treatment outcome, there has been dramatic progress in the understanding of the biology of chronic lymphocytic leukemia (CLL) and a number of new prognostic factors have been identified. ${ }^{1}$ Although there is strong evidence linking prognosis with biological markers, it is by no means defined how these factors should be used in the management of patients with CLL. In addition, before being incorporated into daily practice these markers must be standardized and validated in large prospective trials. ${ }^{2}$

The staging systems defined by Rai et al. ${ }^{3}$ and Binet et al. ${ }^{4}$ were based on their prognostic significance and the stage of disease still remains the most important prognostic indicator in CLL. However, clinical staging systems were developed in the late 1970s and over the years stage of disease has lost some of its usefulness, since most patients are now being diagnosed in an early stage, reflecting a broader use of routine blood evaluations. ${ }^{5.7}$ As a logical consequence, there is still a need for a simple and reliable method of risk stratification suitable for all patients with CLL.

In line with efforts that led to the development of reliable and widely available prognostic systems in multiple myeloma and follicular lymphoma, ${ }^{8,9}$ Wierda et al..$^{10}$ proposed a new prognostic index for patients with CLL. The model predicting overall survival was constructed using six factors (i.e., age, absolute lymphocyte count, gender, $\beta 2$-microglobulin concentration, Rai clinical stage and number of involved lymph node regions) that were independently associated with patients' survival. ${ }^{10}$ This new prognostic index was recently validated in an independent series of CLL patients observed at the Mayo Clinic. ${ }^{11}$ Furthermore, the utility of the index was extended by the demonstration that its value was retained when applied to Rai stage 0 patients, in whom it could be used to predict time to treatment. ${ }^{11}$

In order to determine the utility of the score in predicting the time to first treatment (TFT), we analyzed the information contained in an observational CLL database run by GIMEMA (Gruppo Italiano Malattie EMatologiche dell'Adulto). ${ }^{12}$

\section{Design and Methods}

\section{Patients}

The GIMEMA CLL database includes information on previously untreated CLL patients in Binet stage A whose diagnosis was immunologically confirmed $\left(\mathrm{CD}^{+} / \mathrm{SmIg}\right.$ weak) and who were observed at different GIMEMA primary hematology centers during the period 1991-2000. All patients were treated outside of therapeutic protocols according to a "wait and see" policy. Overall, data from 1158 patients from 25 centers were entered into a preliminary working file. Twenty patients $(1.7 \%)$ were excluded because of inadequate follow-up.

Data management and analyses were performed in accordance with the ethical guidelines of the GIMEMA Review Board and the tenets of the Declaration of Helsinki. The study was also evaluated and approved by the ethical committee of the Pugliese-Ciaccio Hospital, Catanzaro.

Information regarding five parameters, age, gender, Rai stage, absolute peripheral blood lymphocytosis and number of lymph node sites involved, was available for all 1158 patients, while $\beta 2$ microglobulin levels were available for only 310 patients. The characteristics of the five former parameters were, however, the same for patients with and without $\beta 2$-microglobulin data. This was also the case when the probability of remaining free from therapy at 5 years was evaluated for patients with and without available $\beta 2$-microglobulin data $(80 \%$ versus $78 \%$, respectively; $P=0.07)$.

\section{Indication for therapy}

Active, therapy-requiring disease was defined by the presence of at least one of the following criteria: ${ }^{13}$ (i) evidence of progressive marrow failure, manifested by the development or worsening of anemia and/or thrombocytopenia; (ii) massive (i.e., $>6 \mathrm{~cm}$ below the left costal margin) or progressive or symptomatic splenomegaly; (iii) massive lymph nodes (i.e., $>10 \mathrm{~cm}$ in the longest diameter) or progressive or symptomatic lymphadenopathy; (iv) progressive lymphocytosis with an increase of more than $50 \%$ over a 2 -month period, or a lymphocyte doubling time (LDT) of less than 6 months; (v) autoimmune anemia and/or thrombocytopenia poorly responsive to corticosteroids or other standard therapy; and (vi) unintentional weight loss of $10 \%$ or more within the previous 6 months or significant fatigue (i.e., Eastern Cooperative Oncology Group Performance Score of 2 or worse; unable to work or to perform usual activities) or fever higher than $38.0^{\circ} \mathrm{C}$ for 2 or more weeks without other evidence of infections or night sweats for more than 1 month without evidence of infection.

The absolute lymphocyte count was not used as the sole indicator for treatment.

\section{Nomogram and prognostic index scores}

Age, gender, absolute lymphocyte count, $\beta 2$-microglobulin, Rai stage and number of lymph node regions involved were used to calculate the prognostic index score according to the method proposed by Wierda et al. ${ }^{10}$ Since only Binet stage A patients were included, Rai substages were dichotomized as follows: Rai 0 versus Rai I-II (Table 1).

The nomogram score was also calculated using the published formula:

Total score $=-12.5+[1.25 \times$ age $]+[4.32 \times \beta 2$-microglobulin $]+$ $\left[8.62 \times\right.$ (absolute lymphocyte count $\left.\left.\times 10^{9} / \mathrm{L} / 100\right)\right]+[7.34 \times \mathrm{I}(\mathrm{sex}=$ male $)]+[11.00 \times$ I (Rai stage $=$ III or IV) $]+[10.84 \times$ I $($ lymph nodes $=3)]$, where I is the indicator function equal to 1 if the condition in parenthesis is met and 0 if not.

\section{Statistical analysis}

Estimates of TFT were calculated using the Kaplan-Meier method. Likelihood ratio tests were used to analyze the effects of individual factors, either univariately or jointly. Hazard ratios (HR) and confidence intervals (CI) for these ratios were calculated from the Cox models. In both univariate and multivariate analysis continuous variables such as age, absolute lymphocyte count and $\beta 2$ microglobulin were stratified, as proposed by Wierda et al., ${ }^{10}$ as follows: (i) age (years): less than 50, 50-65, more than 65; (ii) absolute lymphocyte count $\left(\times 10^{9} / \mathrm{L}\right)$ : less than $20,20-50$, more than 50 ; c) $\beta 2$-microglobulin ( $\mathrm{mg} / \mathrm{L})$ : less than the upper limit of normal (ULN)(i.e., $1.8 \mathrm{mg} / \mathrm{L}$ ), 1-2 times the ULN, more than two times the ULN. The recursive partitioning (RPART) model was used to search for appropriate cut-off points of the score of the prognostic index and to determine how best to split patients in Binet stage $\mathrm{A}$ into different subgroups. 


\section{Results}

\section{Patients' characteristics}

The main characteristics of the 310 stage A patients for whom complete data were available for the six parameters used to calculate the newly proposed index score are shown in Table 2. The male to female ratio was 1.27 and the median age at diagnosis was 64 years, with $49 \%$ of patients aged more than 65 years and $30.3 \%$ aged more than 70 years. The majority of patients had Rai stage 0 disease $(67 \%)$ and virtually all were local, non-referred patients, first diagnosed and then managed at different primary Italian hematology centers. The 310 patients were followed for a total of 1,303 person-years (median, 41.5 months; range, 3-199 months) during which 51 required therapy. The probability of remaining free from therapy was $78.8 \%$ at 5 years and $59 \%$ at 10 years, with no plateau reached (Figure 1).

\section{Individual factors and multivariate time to first treatment analysis}

The correlation between the six factors included in the prognostic score described by Wierda et al. ${ }^{10}$ and the TFT is presented in Table 3. Among the baseline factors evaluated with respect to TFT, the following were associated with a more unfavorable clinical outcome: male gender $(P=0.007)$, increased concentration of $\beta 2$-microglobulin $(P=0.0008)$, Rai substage I-II $(P=0.02)$ and higher absolute lymphocyte count $(P=0.0007)$. Associations were not found for age $(P=0.97)$ and number of involved lymph node groups $(P=0.68)$.

Multivariate regression analysis was carried out to assess the relationship between independent variables and TFT in a Cox proportional hazards model. Gender $(P=0.01), \beta 2$ microglobulin level $(P=0.002)$ and absolute lymphocyte count $(P=0.01)$ were found to be independently associated with TFT in this analysis (Table 4). Interestingly, the hazard ratio higher than 2 found for all these variables qualifies the analysis as highly discriminating.

\section{Nomogram score}

For each patient we calculated the nomogram score using the formula proposed by Wierda et al..$^{10}$ The median nomogram score for the 310 Binet stage A patients was 80

Table 1. Prognostic index based on the presence of risk factors.

\begin{tabular}{lcccc} 
Characteristic & \multicolumn{4}{c}{ Point contribution } \\
& $\mathbf{0}$ & $\mathbf{1}$ & $\mathbf{2}$ & $\mathbf{3}$ \\
Age, years & - & $<50$ & $50-65$ & $>65$ \\
$\beta_{2} \mathrm{M}, \mathrm{mg} / \mathrm{L}$ & $<\mathrm{ULN}$ & $1-2 \times$ ULN & $>2 \times$ ULN & - \\
\hline $\mathrm{ALC}, \times 10^{9} / \mathrm{L}$ & $<20$ & $20-50$ & $>50$ & - \\
Gender & Female & Male & - & - \\
\hline Rai stage* & 0 & I-II & - & - \\
N. of involved & $0-2$ & $\geq 3$ & - & - \\
nodal groups & & & &
\end{tabular}

$\beta_{2}$ : $\beta_{2}$-microglobulin; ULN: upper limit of normal; ALC: absolute lymphocyte count. The patient's total score was obtained by adding up the scores for the six components. Adapted from Wierda et al. ${ }^{10}$ * Since only Binet stage A patients were included in this analysis, Rai substages were dichotomized as follows: Rai 0 versus Rai I-II. (range, 34-120), which was similar to the median score reported by both the M.D. Anderson Cancer Center (median, 82.9) and the Mayo Clinic (median, 83.9). The median nomogram score enabled the patients to be stratified into two groups with different TFT $\left(\chi^{2}=18.73\right.$; $P<0.0001 ; \mathrm{HR}=3.95 ; 95 \% \mathrm{CI}, 3.95-5.47$ ) (Figure 2).

\section{M.D. Anderson Cancer Center prognostic index score}

Using the method proposed by Wierda et al. ${ }^{10}$ we calculated the prognostic index score for the 310 Binet stage $\mathrm{A}$ patients. Given the cohort of patients considered in this study (i.e., all with Binet stage A disease), no patient had a score higher than 7 , necessary for inclusion in the highrisk group. Therefore, according to the prognostic index, $47 \%$ of patients met the criteria for low risk (score $0-3$ ) and $53 \%$ the criteria for intermediate risk (score 4-7).

The new prognostic index enabled the Binet stage A patients to be divided into two subgroups that differed with respect to TFT. The estimated median TFT was not reached for the low risk group, while it was 111 months for patients in the intermediate risk category $\left(\chi^{2}=8.50\right.$; $P=0.003$; HR $=2.38 ; 95 \% \mathrm{CI}, 1.29-3.72$ ) (Figure 3). Even though our focus was the TFT, the index scores of our cohort were related to overall survival. The actuarial survival probability at 5 years was $99.3 \%$ and $88.2 \%$ for patients who scored $0-3$ and $4-7$, respectively $(P=0.0005)$.

Since the stratification according to the Rai system, used as a comparison, showed a lower discriminating power

Table 2. Patients' characteristics $(n=310)$.

\begin{tabular}{lc}
\hline Characteristic & Number (\%) \\
\hline Age at diagnosis (years) & \\
\hline$<50$ & $27(8.7)$ \\
$50-69$ & $77(24.8)$ \\
$60-69$ & $111(35.8)$ \\
$70-79$ & $78(25.1)$ \\
$>80$ & $17(5.4)$ \\
Gender & \\
\hline Male & $174(56.1)$ \\
Female & $136(43.8)$ \\
Rai stage at diagnosis & \\
\hline 0 & $208(67)$ \\
I-II & $102(32.9)$ \\
Absolute lymphocyte count $\left(\times 10^{9} / \mathrm{L}\right)$ & \\
\hline$<20$ & $245(78)$ \\
$20-50$ & $54(17.4)$ \\
$>50$ & $11(3.5)$ \\
Serum $\beta_{2}$-microglobulin $(\mathrm{mg} / \mathrm{L})$ & $157(50.6)$ \\
\hline$<$ ULN* & $132(42.5)$ \\
$1-2 \mathrm{x}$ ULN & $11(3.5)$ \\
$>2 \times$ ULN & \\
\hline Number of lymph node sites involved & $290(93.5)$ \\
\hline $0-2$ & $20(6.4)$ \\
\hline or more &
\end{tabular}

${ }^{*} U L N$ indicates upper limit of normal (i.e., $1.8 \mathrm{mg} / \mathrm{L}$ ). 
$\left(\chi^{2}=4.22 ; P=0.03 ; \mathrm{HR}=1.86 ; 95 \% \mathrm{CI}, 1.02-3.21\right)$, we wondered whether the new prognostic index could add prognostic information to that afforded by Rai staging. All patients in Rai stage I-II fulfilled the criteria for an intermediate risk score according to Wierda et al. ${ }^{10}$ and were, therefore, considered not suitable for this analysis. As far as concerns Rai stage 0 , differences in TFT were observed among patients according to whether they were classified as being at low or intermediate risk by the prognostic index $\left(\chi^{2}=18.80 ; P<0.0001 ; \mathrm{HR}=2.79 ; 95 \% \mathrm{CI}, 3.92-37.4\right)$.

The prognostic index proposed by Wierda et al. ${ }^{10}$ was originally derived from a database collecting information on CLL patients observed at a reference academic center; these patients had more advanced disease $(P<0.0001)$ and were younger $(P<0.0001)$ than our cohort of patients (Table 5). With this in mind, we wondered whether different cut-off scores would predict patients' TFT better in the GIMEMA series, which consisted of local, non-referred patients with early disease studied at presentation. We, therefore, applied an optimal cut-off score search to determine how best to split Binet stage A patients into different subgroups. According to the RPART model, we were able to build a classification tree that identified three subsets of patients who scores were: 0-2 (low risk), 3-4 (intermediate risk) and 5-7 (high risk) (Figure 4). When applied to our set of Binet stage A patients, the modified prognostic score documented a clear-cut separation between different groups. As shown in Figure 5, the probability of remaining free from therapy at 5 years was $100 \%$ in the low risk group, $81.2 \%$ in the intermediate risk group and $61.3 \%$ in the high risk group $\left(\chi^{2}\right.$ for trend=16.87; $\left.\mathrm{df}=1 ; P<0.0001\right)$.

\section{Discussion}

Wierda et al. ${ }^{10}$ developed a prognostic index, based on widely available clinical and laboratory features, to predict survival among patients with previously untreated CLL. The validity and reproducibility of this index was recently confirmed in an independent series of patients followed at the Mayo Clinic. ${ }^{11}$ Our analysis based on an observational CLL multicenter database assessed the utility of the prognostic index proposed by Wierda et al. ${ }^{10}$ to predict TFT in patients with early disease.

Table 3. Univariate Cox proportional hazard model for time to first treatment.

\begin{tabular}{|c|c|c|c|}
\hline Variable & Hazard ratio & $\begin{array}{l}95 \% \text { Confidence } \\
\text { interval }\end{array}$ & Pvalue \\
\hline Gender & 2.25 & $1.23-4.11$ & 0.007 \\
\hline Rai stage 0 vs I-II & 1.89 & $1.07-3.33$ & 0.02 \\
\hline $\begin{array}{l}\text { Absolute lymphocyte } \\
\text { count* }\end{array}$ & 3.54 & $0.59-7.86$ & 0.0009 \\
\hline$\beta_{2}$-microglobulin* & 4.23 & $2.40-7.48$ & $<0.00001$ \\
\hline Age* & 0.60 & $0.20-3.40$ & 0.97 \\
\hline $\begin{array}{l}\text { N. lymph node groups } \\
\text { involved: } \leq 2 \text { vs. } \geq 3\end{array}$ & 0.75 & $0.18-3.08$ & 0.68 \\
\hline
\end{tabular}

*The statistical analysis was carried out after stratifying patients, as proposed by Wierda et al. ${ }^{10}$, into the following groups: (a) age (years): $<50,50-65,>65$; (b) absolute

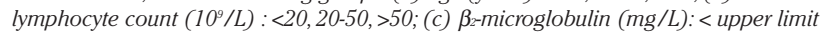
of normal $(U L N), 1-2 U L N,>2 x U L N$.

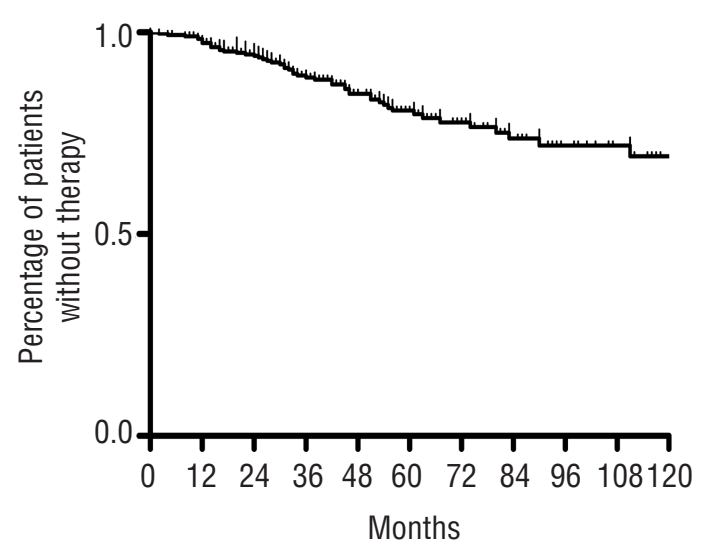

Figure 1. Kaplan-Meier estimate of the time to first treatment of 310 patients with Binet stage A CLL.

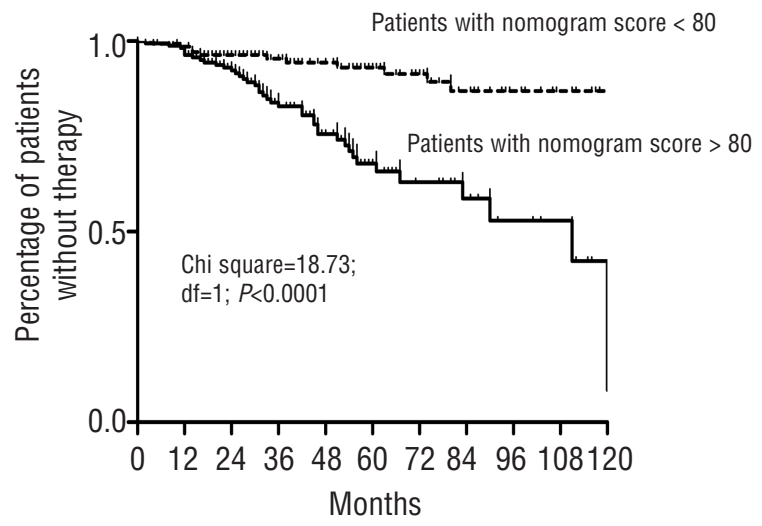

Figure 2. Kaplan-Meier estimate of the time to first treatment of Binet stage A patients stratified according to the median value obtained by the M.D. Anderson Cancer Center nomogram score. ${ }^{9}$

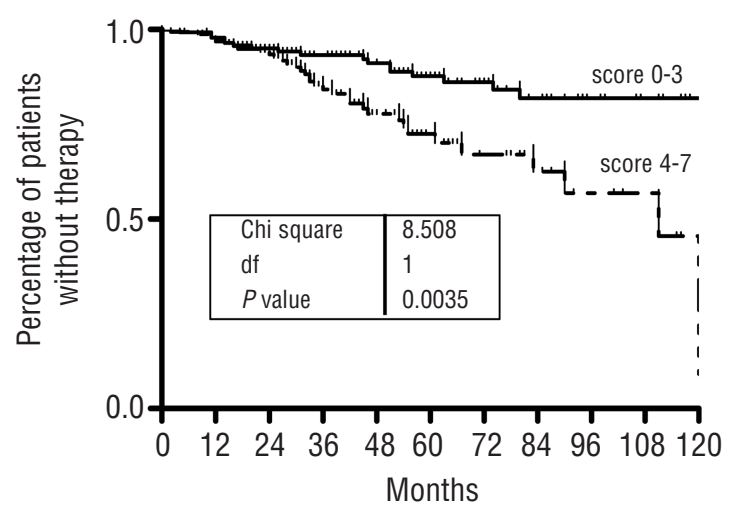

Figure 3. Kaplan-Meier estimate of the time to first treatment by prognostic index category in patients with Binet stage A CLL. 
The original prognostic index was derived from a database that included data on CLL cases observed at a reference academic center; the patients, therefore, had more advanced disease than our series, which consisted of community-based Binet stage A patients whose data were recorded at presentation. These differences between the cohorts of patients led to some revisions of the original scoring system proposed by Wierda et al. ${ }^{10}$ Consequently, we reassigned the points for disease stage according to Rai et $\mathrm{al}^{3}$ and the point groupings were reassessed on the basis of a decision tree analysis.

This study was made possible by the fact that in Italy patients with lymphocytosis are referred to hematology centers. ${ }^{12}$ This allows a horizontal long-term observational follow-up of patients with CLL from early diagnosis that is representative of the natural course of the disease. The same does not apply for the studies recently reported by the two academic referral centers in the USA. In detail, $25 \%$ of the patients evaluated at the M.D. Anderson Cancer Center received treatment within 60 days of first observation, while $65.7 \%$ of patients evaluated at the Mayo Clinic could be considered referred. ${ }^{10,11}$ Consequently, different degrees of both lead and length time bias affect the results of these previously published studies when time-related end-points such as overall survival and TFT are evaluated.

Identifying prognostic factors and developing models that predict clinical end-points are of great importance in order to understand the disease better and to be able to provide more accurate information to patients. Among the end-points that can be studied in prognostic analyses, we decided to measure outcome in terms of TFT, which appears more suitable than overall survival for patients with early CLL. TFT does not reflect competing risks between successive relapses, histological transformation, deaths in remission or the impact of new therapies. Consequently, the fact that some of the variables in the index do not correlate with TFT in univariate or multivariate analyses does not mean that the associations reported

Classification tree for all 310 Binet stage A patients

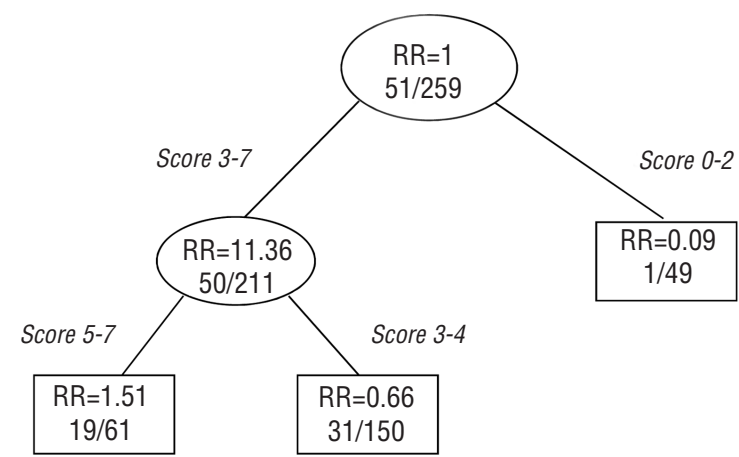

Figure 4. Classification tree built according to recursive partitioning (RPART). The number of patients who required therapy in each group is shown first and then the total number of patients. RR indicates the relative risk. for these variables by the M.D. Anderson Cancer Center do not hold. In fact, the original analysis reported exclusively on overall survival with no data on TFT, while the current analysis does the reverse.

Several paradigms of CLL have changed in the last 30 years. First of all, a shift toward diagnosis at an early stage has now been recognized; accordingly, it is inappropriate to reassure all patients with early-stage disease that they should not be concerned about their disease..$^{5-7}$ Patients with early-stage CLL are a very heterogeneous population

Table 4. Multivariate Cox proportional hazard model for time to first treatment.

\begin{tabular}{lccc} 
Variable & Hazard ratio & $\begin{array}{c}95 \% \text { Confidence } \\
\text { Interval }\end{array}$ & P value \\
Gender & 2.23 & $1.22-4.09$ & 0.01 \\
$\beta_{2 \text { microglobulin }}$ & 2.46 & $1.40-4.04$ & 0.002 \\
\hline $\begin{array}{l}\text { Absolute lymphocyte } \\
\text { count }\end{array}$ & 3.09 & $1.39-6.87$ & 0.006 \\
\hline
\end{tabular}

Table 5. Comparison between demographic characteristics of the M.D. Anderson Cancer Center (MDACC) series and the GIMEMA series.

\begin{tabular}{lccc} 
Male/Female & MDACC series* & GIMEMA series & P value \\
Age & $1029 / 645$ & $174 / 136$ & 0.07 \\
\hline$<50$ years & $395(23.5 \%)$ & $28(9.0 \%)$ & \\
$50-65$ years & $838(50.0 \%)$ & $143(46.1 \%)$ & $<0.0001$ \\
$>65$ years & $441(26.3 \%)$ & $139(44.8 \%)$ & \\
Rai stage & & & \\
\hline 0 & $469(28.1 \%)$ & $208(67.0 \%)$ & \\
I-II & $974(58.3 \%)$ & $102(32.9 \%)$ & $<0.0001$ \\
III-IV & $220(11.9 \%)$ & Not included & \\
\hline
\end{tabular}

*Wierda et al. ${ }^{10}$ 
with respect to clinical outcome ${ }^{14}$ and risk stratification is, therefore, particularly important in this subset of patients, currently the most numerous, in order to provide individualised and tailored follow-up.

Recently identified prognostic factors - i.e., mutational status of the IGVH gene regions, ZAP-70 or CD-38 expression, cytogenetic abnormalities, and p53 mutations - can be used to stratify asymptomatic patients with early disease into low-risk, intermediate-risk and high-risk categories. ${ }^{14-21}$ As shown, in the present study the prognostic index proposed by Wierda et al. ${ }^{10}$ which is based on clinical and basic laboratory characteristics, has proven to be a powerful risk stratification system also for patients with early CLL. Nonetheless, we believe that refinement of this system, through the addition of molecular and biological parameters, will contribute to improve its prognostic accuracy further.

In order to exclude a possible bias due to the fact that $\beta 2$-microglobulin values at the time of diagnosis were available for only 310 of the 1158 patients, the overall characteristics of the individual parameters included in the score index, as well as TFT, were analyzed for patients with and without available $\beta 2$-microglobulin data and no differences were observed.

Finally, our study has a number of important strengths. The individuals studied were from a well-defined cohort of CLL patients participating in observational trials. All patients studied had early stage disease at the entry into the study and thus represent the group of patients for whom prognostic instruments are most needed. Moreover, our effort to develop a revised scoring method meets the need to separate Binet stage A patients into different prognostic groups, in order to devise individualized and tailored follow-up during the treatment-free period.

\section{Authorship and Disclosures}

SM and RF were the principal investigators, designed the study, interpreted the data and wrote the article; DG performed the statistical analysis; SM, MFR, VC, FL, BR, $A C$, and VL recruited the patients.

The authors reported no potential conflicts of interest.

\section{References}

1. Caligaris-Cappio F, Ghia P. Novel insights in chronic lymphocytic leukemia: are we getting closer to understanding the pathogenesis of the disease? J Clin Oncol. 2008; 26(27):4497-503.

2. Moreno C, Montserrat E. New prognostic markers in chronic lymphocytic leukemia. Blood Rev. 2008; 22(4):211-9.

3. Rai KR, Sawitsky A, Cronkite EP, Chanana $\mathrm{AD}$, Levy RN, Pasternack BS. Clinical staging of chronic lymphocytic leukemia. Blood. 1975; 46(2):219-34.

4. Binet IL, Auquier A, Dighiero G, Chastang $\mathrm{C}$, Piguet $\mathrm{H}$, Goasguen J, et al. A new prognostic classification of chronic lymphocytic leukemia derived from a multivariate survival analysis. Cancer. 1981;48(1):198-206.

5. Rozman C, Bosch F, Montserrat E. Chronic lymphocytic leukemia: a changing natural history? Leukemia. 1997; 11(6): 775-8.

6. Molica S, Levato D. What is changing in the natural history of chronic lymphocytic leukemia? Haematologica. 2001;86(1):8-12.

7. Abrisqueta P, Pereira A, Rozman C, Aymerich M, Giné E, Moreno C, et al. Improving survival in patients with chronic lymphocytic leukemia (1980-2008): the Hospital Clinic of Barcelona experience. Blood. 2009;114(10):2044-50.

8. Greipp PR, San Miguel J, Durie BG, Crowley JJ, Barlogie B, Bladé J, et al International staging system for multiple myeloma. J Clin Oncol. 2005;23(15):341220.
9. Solal-Céligny P, Roy P, Colombat P, White J, Armitage JO, Arranz-Saez R, et al. Follicular lymphoma international prognostic index. Blood. 2004;104(5):1258-65.

10. Wierda WG, O'Brien S, Wang X, Faderl S, Ferrajoli A, Do KA, et al. Prognostic nomogram and index for overall survival in previously untreated patients with chronic lymphocytic leukemia. Blood. 2007;109(11):4679-85.

11. Shanafelt TD, Jenkins G, Call TG, Zent CS, Slager S, Bowen DA, et al. Validation of a new prognostic index for patients with chronic lymphocytic leukemia. Cancer. 2009;115(2):363-72.

12. Molica S, Mauro FR, Callea V, Gentile M, Giannarelli D, Lopez M, et al. A genderbased score system predicts the clinical outcome of patients with early B-cell chronic lymphocytic leukemia. Leuk Lymphoma. 2005;46(4):553-60.

13. Hallek M, Cheson BD, Catovsky D, Caligaris-Cappio F, Dighiero G, Döhner H, et al. Guidelines for the diagnosis and treatment of chronic lymphocytic leukemia: a report from the International Workshop on Chronic Lymphocytic Leukemia updating the National Cancer Institute-Working Group 1996 guidelines. Blood. 2008;111(12):5446-56

14. Shanafelt TD, Byrd JC, Call TG, Zent CS, Kay NE. Narrative review: initial management of newly diagnosed, early-stage, chronic lymphocytic leukemia. Ann Intern Med. 2006;145(6):435-47.

15. Gribben JG. Molecular profiling in CLL. Hematology Am Soc Hematol Educ
Program. 2008:444-9

16. Hamblin TJ, Davis Z, Gardiner A, Oscier DG, Stevenson FK. Unmutated $\operatorname{IgV}(\mathrm{H})$ genes are associated with a more aggressive form of chronic lymphocytic leukemia. Blood. 1999;94(6):1848-54.

17. Damle RN, Wasil T, Fais F, Ghiotto F, Valetto A, Allen SL, et al. Ig V gene mutation status and CD38 expression as novel prognostic indicators in chronic lymphocytic leukemia. Blood. 1999;94(6):1840-7.

18. Crespo M, Bosch F, Villamor N, Bellosillo B, Colomer D, Rozman M, et al. ZAP-70 expression as a surrogate for immunoglobulin-variable-region mutations in chronic lymphocytic leukemia. N Engl J Med. 2003;348(18):1764-75.

19. Rassenti LZ, Huynh L, Toy TL, Chen L, Keating MJ, Gribben JG, et al. ZAP-70 compared with immunoglobulin heavy-chain gene mutation status as a predictor of disease progression in chronic lymphocytic leukemia. N Engl J Med. 2004;351(9): 893901.

20. Hamblin TJ, Orchard JA, Ibbotson RE, Davis Z, Thomas PW, Stevenson FK, et al. CD38 expression and immunoglobulin variable region mutations are independent prognostic variables in chronic lymphocytic leukemia, but CD38 expression may vary during the course of the disease. Blood. 2002; 99(3):1023-9.

21. Döhner H, Stilgenbauer S, Benner A, Leupolt E, Kröber A, Bullinger L, et al. Genomic aberrations and survival in chronic lymphocytic leukemia. N Engl J Med. 2000; 343(26):1910-6. 